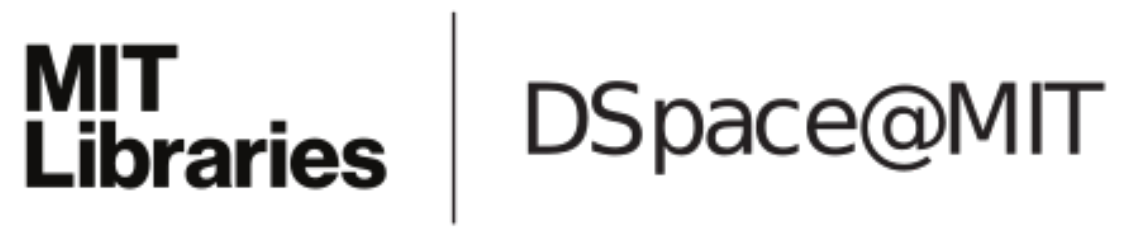

\author{
MIT Open Access Articles
}

Electrical Conductivity in a Porous, Cubic Rare-Earth Catecholate

The MIT Faculty has made this article openly available. Please share how this access benefits you. Your story matters.

Citation: Skorupskii, Grigorii and Mircea Dincă, "Electrical Conductivity in a Porous, Cubic RareEarth Catecholate." Journal of the American Chemical Society 142, 15 (March 2020): 6920-24 doi. 10.1021/jacs.0c01713 @2020 Authors

As Published: https://dx.doi.org/10.1021/jacs.0c01713

Publisher: American Chemical Society (ACS)

Persistent URL: https://hdl.handle.net/1721.1/125534

Version: Final published version: final published article, as it appeared in a journal, conference proceedings, or other formally published context

Terms of use: Creative Commons Attribution 4.0 International license 


\title{
Electrical Conductivity in a Porous, Cubic Rare-Earth Catecholate
}

\author{
Grigorii Skorupskii and Mircea Dincä*
}

Cite This: J. Am. Chem. Soc. 2020, 142, 6920-6924

Read Online

ABSTRACT: Electrically conductive metal-organic frameworks (MOFs) provide a rare example of porous materials that can efficiently transport electrical current, a combination that is favorable for a variety of technological applications. The vast majority of such MOFs are highly anisotropic in both their structures and properties: Only two electrically conductive MOFs reported to date exhibit cubic structures that enable isotropic charge transport. Here we report a new family of intrinsically porous frameworks made from rare-earth nitrates and hexahydroxytriphenylene. The materials feature a novel hexanuclear secondary building unit and form cubic, porous, and intrinsically conductive structures, with electrical conductivities reaching $10^{-5} \mathrm{~S} / \mathrm{cm}$ and surface areas of up to 780 $\mathrm{m}^{2} / \mathrm{g}$. By expanding the list of MOFs with isotropic charge transport, these results will help us to improve our understanding of design strategies for porous electronic materials.

$\mathrm{R}$ ecent advances in electrically conductive metal-organic frameworks (MOFs) significantly expanded the range of applications for this broad class of hybrid organic-inorganic materials, achieving important milestones in charge-storage materials, ${ }^{1,2}$ electrochemical catalysts, ${ }^{3,4}$ and chemiresistive sensors. ${ }^{5}$ Much work focuses on expanding the library of electrically conductive porous MOFs, yet the vast majority of such materials exhibit charge transport that occurs preferentially along a low-dimensional pathway, such as a 1D metalligand chain, ${ }^{6,7}$ a $\pi-\pi$ stacked column, ${ }^{8}$ or a $\pi$-conjugated sheet. ${ }^{9}$ Although anisotropic transport may be desired in certain instances, as evidenced by the rapidly developing fields of low-dimensional materials such as graphene ${ }^{10}$ and silicon nanowires, ${ }^{11}$ it is of significant fundamental interest to develop conductive MOFs with isotropic charge transport. ${ }^{12}$ Remarkably, to date, only two ${ }^{13,14}$ MOFs with cubic symmetry have been reported to show intrinsic (non-guest-based) electrical conductivity.

The highest conductivities to date are observed in MOFs based on ligands with catechol- and semiquinone-derived functional groups, such as hexaaminotriphenylene, ${ }^{9,15}$ and hexahydroxytriphenylene (HHTP), ${ }^{3,16}$ among others. ${ }^{17-26} \mathrm{~A}$ number of factors makes these ligands especially viable for making electrically conductive frameworks, including the strong $\pi-\mathrm{d}$ conjugation between the metal and the ligand, the ligands' propensity for $\pi-\pi$ stacking, and, crucially, their redox activity: The oxidation of the catechol moieties forms semiquinoid radicals that serve as the charge carriers in the materials. $^{27}$ It is therefore unsurprising that of the two conductive MOFs with cubic symmetry, one is based on a ligand belonging to this class, dihydroxybenzoquinone (DHBQ). The cubic framework $\left[\mathrm{Fe}_{2}(\mathrm{DHBQ})_{3}\right]\left[\mathrm{Bu}_{4} \mathrm{~N}\right]_{2}$ shows an impressive conductivity of $0.16 \mathrm{~S} / \mathrm{cm} .{ }^{14}$ This value is controllable through postsynthetic redox chemistry, where the radical semiquinoid linkers can be reduced to their closedshell catechol form to lower the free carrier concentration. This framework, however, is nonporous due to the bulky charge- compensating tetrabutylammonium cations blocking the small pores. Nevertheless, partly inspired by these results, we turned to the larger, chemically related HHTP ligand to target a potentially porous, isotropically conductive MOF.

The reaction of concentrated solutions $(>0.5 \mathrm{M})$ of hydrated rare-earth nitrates $\mathrm{M}\left(\mathrm{NO}_{3}\right)_{3} \cdot n \mathrm{H}_{2} \mathrm{O}(\mathrm{M}=\mathrm{Y}, \mathrm{Eu}$, La) with HHTP in a mixture of water and $N, N^{\prime}$ dimethylimidazolidinone (DMI) at temperatures above 150 ${ }^{\circ} \mathrm{C}$ yields large octahedral yellow crystals (Figure S11) of $\left[\mathrm{M}_{6}\left(\mu_{6}-\mathrm{NO}_{3}\right)(\text { HOTP })_{2}\right]^{5+}\left(\mathbf{M}_{6}\right.$ HOTP $_{2}, \mathbf{M}=\mathrm{Y}, \mathrm{Eu} ;$ HOTP $=$ $2,3,6,7,10,11$-hexaoxytriphenylene). The charge balance is provided by five monoanions (hydroxides or nitrates), which correspond to a fully reduced ligand, $\mathrm{HOTP}^{6-}$. This assignment is corroborated by the color of the crystals: The yellowbrown color matches the color of unoxidized, air-free solutions of HHTP. Minor oxidation of HHTP leads to the formation of intensely colored blue or purple species. X-ray diffraction analysis of single crystals revealed that all three compounds crystallize in the cubic space group $F d \overline{3} m$. (See Figure 1 and Tables S2 for structural parameters.) The secondary building units (SBUs) consist of hexanuclear $\mu_{6}$-nitrato catecholate clusters (Figure $2 \mathrm{~b}$ ), wherein the metal ions alternate above and below the plane of the central nitrate ion in a chairlike conformation. Six HHTP ligands surround each secondary building unit (SBU) and connect neighboring SBUs into tetrahedral cages (Figure $2 \mathrm{c}$ ). The cages themselves are connected at the vertices to provide a net with 6- and 3connected nodes and overall spn topology (Figure 2a). The same topology has been previously observed in several MOFs,

Received: February 12, 2020

Published: March 28, 2020 


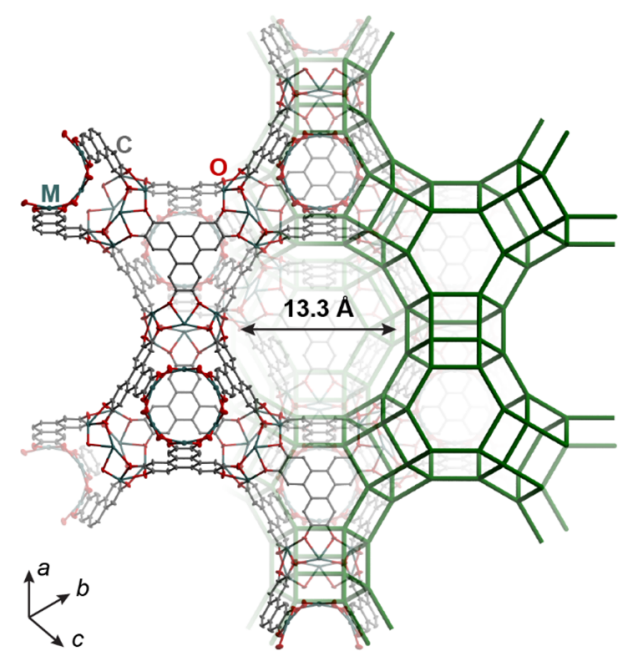

Figure 1. Crystal structure of $\mathbf{M}_{6} \mathrm{HOTP}_{2}(\mathrm{M}=\mathrm{Y}, \mathrm{Eu})$, seen along the (111) crystallographic direction, is similar to the well-known faujasite zeolite structure type (green schematic overlay) and consists of diamondoid cages with permanent solvent-accessible pores that are $\sim 13.3 \AA$ in diameter. Carbon atoms are shown in gray, nitrogen in blue, oxygen in red, and metals in teal. Thermal ellipsoids are shown at the $50 \%$ probability level. Hydrogen atoms, as well as nitrato and aqua ligands, are omitted for clarity.

most notably in MOF- $808,{ }^{28}$ and is homologous to the faujasite zeolite structure type (Figure 1).

The SBU shows significant crystallographic disorder, as may be expected given the coordination flexibility of rare-earth ions. Metal atoms in $\mathbf{E u}_{6} \mathbf{H O T P}_{2}$ and $\mathbf{Y}_{6} \mathrm{HOTP}_{2}$ have similar eightcoordinate environments (Figure S5), with each metal connected to one oxygen atom of the central bridging nitrate, four catecholate oxygen atoms of three different HOTP linkers, and a disordered combination of aqua and nitrato ligands oriented toward the pore or the inner cavity of the tetrahedral cage. Notably, this SBU has not been previously observed in MOFs. The discovery of new SBUs is a driver for expanding the reticular chemistry of MOFs, ${ }^{29}$ yet reports of new inorganic building units whose structural features are conserved across different metals have become increasingly rare. The new SBU is particularly notable because it features catecholate ligands, which thus far have given rise almost exclusively to single-ion SBUs, with very few exceptions. ${ }^{2,30-32}$ As with the earliest SBUs made from carboxylates, the hexanuclear SBU reported here is also a close mimic of a molecular hexalanthanide cluster that has been previously reported (Figure S6). ${ }^{33}$ This similarity reinforces the strategy of targeting high-symmetry clusters known in molecular chemistry as potential SBUs for novel MOFs, a strategy commonly employed in reticular chemistry with more traditional functional groups such as carboxylates and azolates. $^{34}$

Owing to its propensity to $\pi$-stack, HHTP rarely forms $3 \mathrm{D}$ structures. $^{12}$ Indeed, only one other report details the formation of isotropic, 3D MOFs from HHTP. ${ }^{27}$ Instead, this ligand preferably leads to stacked structures ${ }^{16}$ reinforced by close contacts between its extended aromatic cores. Notably, although the 2D sheets and the close contacts in most HHTP-based materials lead to some of the highest electrical conductivities among porous MOFs, ${ }^{35}$ the electrical transport in these materials is anisotropic. In fact, almost all conductive MOFs reported thus far have lower symmetry than cubic and therefore exhibit anisotropic transport. ${ }^{12}$

To probe whether $\mathbf{M}_{6} \mathbf{H O T P} \mathbf{P}_{2}$ represents a rare example of a cubic, electrically conductive MOF, we tested the electrical conductivity of activated crystalline powders with a two-probe apparatus that has been previously described. ${ }^{36}$ The activation of the crystals involved heating under a dynamic vacuum at 95 ${ }^{\circ} \mathrm{C}$ for $18 \mathrm{~h}$. We note that thermogravimetric analysis of assynthesized samples exchanged sequentially with water, methanol, and acetonitrile showed a first significant mass loss
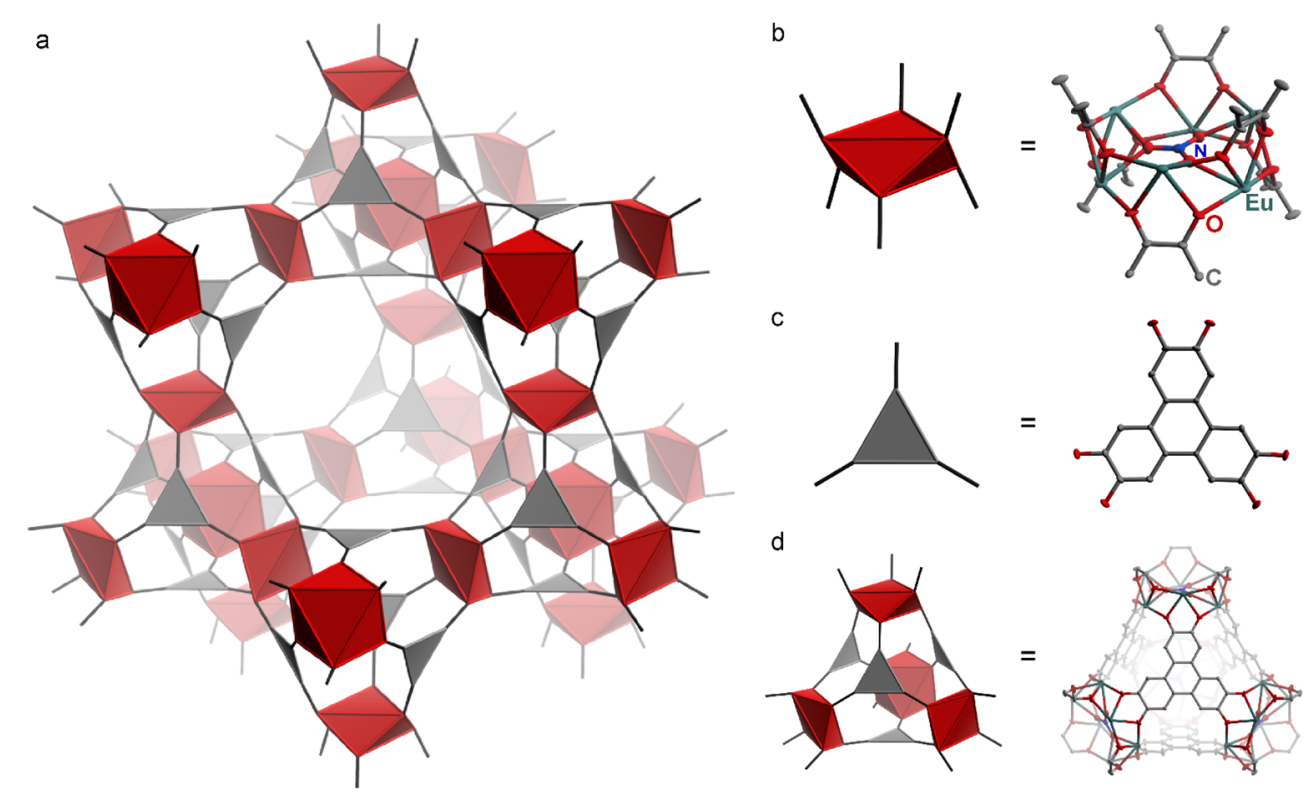

Figure 2. Portions of the crystal structure of $\mathbf{M}_{6} \mathbf{H O T P}{ }_{2}$. (a) The framework is built from 6-connected SBU nodes and 3-connected linker nodes, resulting in the overall spn topology. (b) The SBU consists of six metal atoms bonded by one bridging nitrate ion in the center and connected along the sides with catecholate groups from six linkers. (c) The trigonal HOTP ${ }^{6-}$ connects the SBUs into tetrahedral cages (d). The cavities formed inside the cages appear to be inaccessible to solvent or other guest molecules. Carbon atoms are shown in gray, nitrogen in blue, oxygen in red, and europium in teal. Thermal ellipsoids are shown at the 50\% probability level. Hydrogen atoms are omitted for clarity. 
at $\sim 90{ }^{\circ} \mathrm{C}$ (Figure S1). A representative sample of $\mathbf{Y}_{6} \mathbf{H O T P}_{2}$, activated as previously described, also showed permanent porosity, as indicated by a type $\mathrm{I}_{2}$ adsorption isotherm, which can be fit to the Brunauer-Emmett-Teller (BET) model $^{37}$ to give an apparent surface area of $780 \mathrm{~m}^{2} / \mathrm{g}$. This value is in line with the reported surface area of the topologically equivalent MOF-808 $\left(1140 \mathrm{~m}^{2} / \mathrm{g}\right),{ }^{28}$ which has near-identical unit-cell parameters to those of $\mathbf{Y}_{6} \mathbf{H O T P}_{2}$.

Relevantly, although as-synthesized crystals of $\mathbf{M}_{6} \mathbf{H O T P}$ are yellow, they slowly darken (Figure S12) over time, even under rigorous air-free conditions, likely indicating at least the partial oxidation of catechol moieties in $\mathrm{HOTP}^{6-}$ ligands to semiquinones. This transformation happens over the course of many weeks at room temperature, but heating or exposure to air causes significant acceleration. Although prolonged exposure to air leads to the eventual loss of crystallinity, up to complete amorphization (Figure S8), heating under inert conditions preserves the structure while still accelerating the color change.

We tracked this color change by in situ diffuse reflectance spectroscopy of a powder of $\mathbf{Y}_{6} \mathbf{H O T P}_{2}$ heated to $90{ }^{\circ} \mathrm{C}$ under a dinitrogen atmosphere, as shown in Figure 3. The spectra

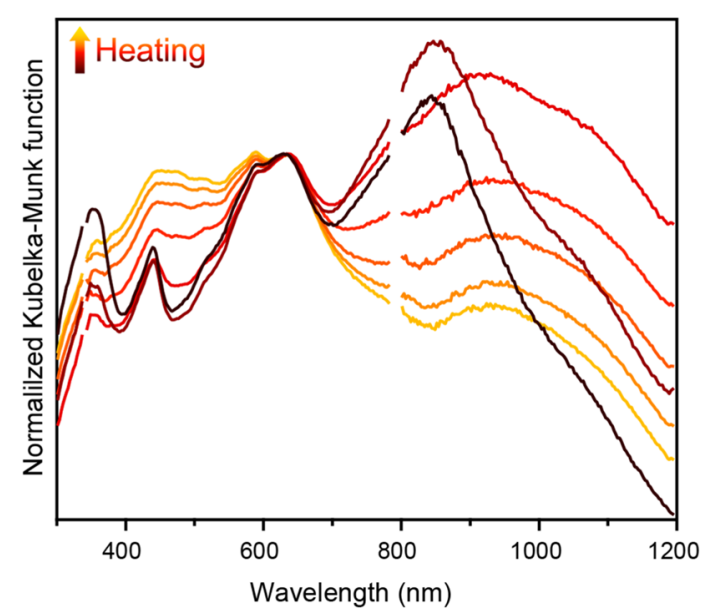

Figure 3. Diffuse reflectance spectra for $\mathbf{Y}_{6} \mathbf{H O T P}_{2}$, showing the evolution of the electronic structure of the material on heating under an inert atmosphere (black, initial spectrum; yellow, final spectrum). The broad band at $1000-1200 \mathrm{~nm}$ is often considered a fingerprint of semiquinoid radicals in chemically related compounds.

closely resemble those reported for chemically similar molecular clusters, such as the trinuclear Co(III) HOTP complex, ${ }^{38}$ as well as the trinuclear $\mathrm{Fe}(\mathrm{III})$ complex with a hexaaminotriphenylene-based ligand. ${ }^{39}$ Importantly, spectral changes observed for $\mathrm{Y}_{6}$ HOTP 2 upon heating also mimic the behavior of the Fe(III) complex upon oxidation. Specifically, the MOF spectra exhibit a decrease in relative intensity of the bands at $300-450 \mathrm{~nm}$ (attributed to $\pi-\pi^{*}$ transitions within the aromatic core) and at $800 \mathrm{~nm}$ (attributed to ligand-tometal charge transfer) as well as the appearance of a new band at $1000-1200 \mathrm{~nm}$, commonly associated with the $\pi-\pi^{*}$ transitions in radical semiquinoid moieties. ${ }^{38,39}$

The spectral changes previously discussed confirm the partial oxidation of the material and the potential formation of free charge carriers that could improve the electrical conductivity. Indeed, both $\mathbf{Y}_{6} \mathrm{HOTP}_{2}$ and $\mathbf{E u}_{6} \mathrm{HOTP}_{2}$ show electrical conductivities on the order of $10^{-6}$ to $10^{-5} \mathrm{~S} / \mathrm{cm}$ (Figure 4) upon activation, across several independently

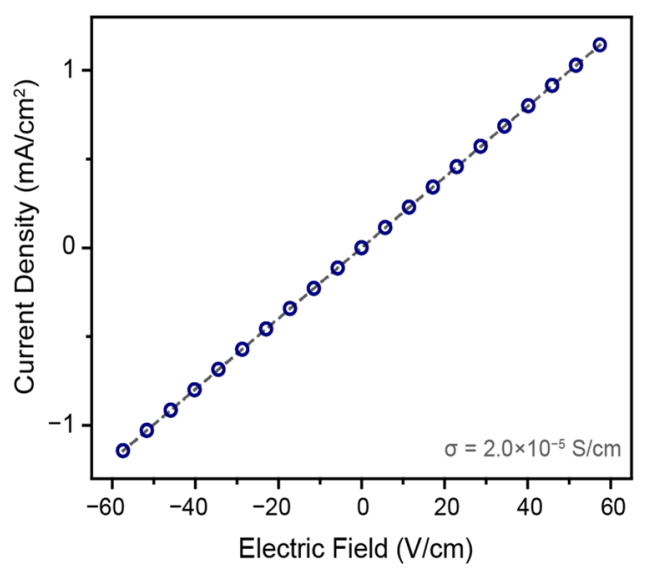

Figure 4. Current-voltage characteristics of $\mathrm{Y}_{6} \mathrm{HOTP}_{2}$, obtained on pressed pellets in a two-probe configuration, showing perfect linear behavior in accordance with Ohm's law. The linear fit of the data (dashed line) reveals the conductivity of this representative device to be $2.0 \times 10^{-5} \mathrm{~S} / \mathrm{cm}$.

synthesized batches. Although not as high as the bulk conductivities of HHTP-derived MOFs that exhibit close HOTP stacking $\left(10^{-4}-10^{-2} \mathrm{~S} / \mathrm{cm}\right),{ }^{3,16}$ these values are remarkable $^{12}$ for $3 \mathrm{D}$, isotropic porous MOFs, where charges are expected to transport with equal efficiency along the three Cartesian coordinates.

It is rare for intrinsic porosity and electrical conductivity to coexist within one material. It is even rarer for such compounds to be structurally isotropic. In this work, we presented a new family of MOFs that combine significant porosity with electrical conductivity in a cubic framework. The relatively high conductivity shown by these materials demonstrates that targeting low-dimensional pathways is not mandatory for achieving efficient charge transport. We hope these results will inspire more detailed studies into the dimensionality of charge transport in this exciting class of materials and eventually lead to a better understanding of how porosity interacts with conductivity.

\section{ASSOCIATED CONTENT}

\section{S1 Supporting Information}

The Supporting Information is available free of charge at https://pubs.acs.org/doi/10.1021/jacs.0c01713.

Additional experimental details and procedures (PDF)

X-ray crystal structure of $\mathbf{E u}_{6} \mathrm{HOTP}_{2}$ (CIF)

X-ray crystal structure of $\mathbf{Y}_{6} \mathrm{HOTP}_{2}$ (CIF)

X-ray crystal structure of $\mathbf{L a}_{6} \mathbf{H O T P}_{2}$ (CIF)

\section{AUTHOR INFORMATION}

\section{Corresponding Author}

Mircea Dincă - Department of Chemistry, Massachusetts Institute of Technology, Cambridge, Massachusetts 02139, United States; 이이.org/0000-0002-1262-1264;

Email: mdinca@mit.edu

\section{Author}

Grigorii Skorupskii - Department of Chemistry, Massachusetts Institute of Technology, Cambridge, Massachusetts 02139, United States

Complete contact information is available at:

https://pubs.acs.org/10.1021/jacs.0c01713 


\section{Author Contributions}

The manuscript was written through contributions of both authors. Both authors have given approval to the final version of the manuscript.

\section{Notes}

The authors declare no competing financial interest.

\section{ACKNOWLEDGMENTS}

This work was supported by the Army Research Office (grant number W911NF-17-1-0174). Single X-ray diffraction data were collected on a Bruker X8 Kappa DUO four-circle diffractometer that was purchased with the help of funding from the National Science Foundation (NSF) under grant number CHE-0946721. We thank Luming Yang, Dr. Maxx Q. Arguilla, and Lilia S. Xie for helpful discussions.

\section{REFERENCES}

(1) Sheberla, D.; Bachman, J. C.; Elias, J. S.; Sun, C.-J.; Shao-Horn, Y.; Dincă, M. Conductive MOF Electrodes for Stable Supercapacitors with High Areal Capacitance. Nat. Mater. 2017, 16, 220-224.

(2) Feng, D.; Lei, T.; Lukatskaya, M. R.; Park, J.; Huang, Z.; Lee, M.; Shaw, L.; Chen, S.; Yakovenko, A. A.; Kulkarni, A.; Xiao, J.; Fredrickson, K.; Tok, J. B.; Zou, X.; Cui, Y.; Bao, Z. Robust and Conductive Two-Dimensional Metal-organic Frameworks with Exceptionally High Volumetric and Areal Capacitance. Nat. Energy 2018, 3, 30-36.

(3) Miner, E. M.; Wang, L.; Dinca, M. Modular $\mathrm{O}_{2}$ Electroreduction Activity in Triphenylene-Based Metal-Organic Frameworks. Chem. Sci. 2018, 9, 6286-6291.

(4) Miner, E. M.; Fukushima, T.; Sheberla, D.; Sun, L.; Surendranath, Y.; Dincă, M. Electrochemical Oxygen Reduction Catalysed by $\mathrm{Ni}_{3}$ (Hexaiminotriphenylene) ${ }_{2}$. Nat. Commun. 2016, 7, 10942.

(5) Stassen, I.; Dou, J.-H.; Hendon, C.; Dincă, M. Chemiresistive Sensing of Ambient $\mathrm{CO}_{2}$ by an Autogenously Hydrated $\mathrm{Cu}_{3}(\text { Hexaiminobenzene })_{2}$ Framework. ACS Cent. Sci. 2019, 5, $1425-1431$.

(6) Xie, L. S.; Sun, L.; Wan, R.; Park, S. S.; DeGayner, J. A.; Hendon, C. H.; Dincă, M. Tunable Mixed-Valence Doping toward Record Electrical Conductivity in a Three-Dimensional Metal-Organic Framework. J. Am. Chem. Soc. 2018, 140, 7411-7414.

(7) Park, J. G.; Aubrey, M. L.; Oktawiec, J.; Chakarawet, K.; Darago, L. E.; Grandjean, F.; Long, G. J.; Long, J. R. Charge Delocalization and Bulk Electronic Conductivity in the Mixed-Valence MetalOrganic Framework $\mathrm{Fe}(1,2,3-\text { Triazolate })_{2}\left(\mathrm{BF}_{4}\right)_{\mathrm{X}}$. J. Am. Chem. Soc. 2018, 140, 8526-8534.

(8) Park, S. S.; Hontz, E. R.; Sun, L.; Hendon, C. H.; Walsh, A.; Van Voorhis, T.; Dincă, M. Cation-Dependent Intrinsic Electrical Conductivity in Isostructural Tetrathiafulvalene-Based Microporous Metal-Organic Frameworks. J. Am. Chem. Soc. 2015, 137, 1774-1777.

(9) Sheberla, D.; Sun, L.; Blood-forsythe, M. A.; Er, S.; Wade, C. R.; Brozek, C. K.; Aspuru-Guzik, A.; Dincă, M. High Electrical Conductivity in $\mathrm{Ni}_{3}(2,3,6,7,10,11 \text {-Hexaiminotriphenylene })_{2}$, a Semiconducting Metal-Organic Graphene Analogue. J. Am. Chem. Soc. 2014, 136, 8859-8862.

(10) Geim, A. K. Graphene: Status and Prospects. Science 2009, 324, $1530-1534$.

(11) Zhang, A.; Zheng, G.; Lieber, C. Nanowires: Building Blocks for Nanoscience and Nanotechnology. In Nanowires: Building Blocks for Nanoscience and Nanotechnology; Springer International Publishing, 2016; p 322.

(12) Xie, L. S.; Skorupskii, G.; Dincă, M. Electrically Conductive Metal-Organic Frameworks. Chem. Rev. 2020, DOI: 10.1021/ acs.chemrev.9b00766.

(13) Gándara, F.; Uribe-Romo, F. J.; Britt, D. K.; Furukawa, H.; Lei, L.; Cheng, R.; Duan, X.; O’Keeffe, M.; Yaghi, O. M. Porous,
Conductive Metal-Triazolates and Their Structural Elucidation by the Charge-Flipping Method. Chem. - Eur. J. 2012, 18, 10595-10601.

(14) Darago, L. E.; Aubrey, M. L.; Yu, C. J.; Gonzalez, M. I.; Long, J. R. Electronic Conductivity, Ferrimagnetic Ordering, and Reductive Insertion Mediated by Organic Mixed-Valence in a Ferric Semiquinoid Metal-Organic Framework. J. Am. Chem. Soc. 2015, 137, 15703-15711.

(15) Campbell, M. G.; Sheberla, D.; Liu, S. F.; Swager, T. M.; Dincă, M. $\mathrm{Cu}_{3}$ (Hexaiminotriphenylene $)_{2}$ : An Electrically Conductive 2D Metal-Organic Framework for Chemiresistive Sensing. Angew. Chem., Int. Ed. 2015, 54, 4349-4352.

(16) Hmadeh, M.; Lu, Z.; Liu, Z.; Gandara, F.; Furukawa, H.; Wan, S.; Augustyn, V.; Chang, R.; Liao, L.; Zhou, F.; Perre, E.; Ozolins, V.; Suenaga, K.; Duan, X.; Dunn, B.; Yamamto, Y.; Terasaki, O.; Yaghi, O. M. New Porous Crystals of Extended Metal-Catecholates. Chem. Mater. 2012, 24, 3511-3513.

(17) Kambe, T.; Sakamoto, R.; Hoshiko, K.; Takada, K.; Miyachi, M.; Ryu, J.-H.; Sasaki, S.; Kim, J.; Nakazato, K.; Takata, M.; Nishihara, H. $\pi$-Conjugated Nickel Bis(Dithiolene) Complex Nanosheet. J. Am. Chem. Soc. 2013, 135, 2462-2465.

(18) Cui, J.; Xu, Z. An Electroactive Porous Network from Covalent Metal-Dithiolene Links. Chem. Commun. 2014, 50, 3986.

(19) Ziebel, M. E.; Gaggioli, C. A.; Turkiewicz, A. B.; Ryu, W.; Gagliardi, L.; Long, J. R. Effects of Covalency on Anionic Redox Chemistry in Semiquinoid-Based Metal-Organic Frameworks. J. Am. Chem. Soc. 2020, 142, 2653-2664.

(20) Jeon, I.-R.; Negru, B.; Van Duyne, R. P.; Harris, T. D. A 2D Semiquinone Radical-Containing Microporous Magnet with SolventInduced Switching from $T_{\mathrm{c}}=26$ to $80 \mathrm{~K}$. J. Am. Chem. Soc. 2015, 137, 15699-15702.

(21) Jiang, Q.; Xiong, P.; Liu, J.; Xie, Z.; Wang, Q.; Yang, X.; Hu, E.; Cao, Y.; Sun, J.; Xu, Y.; Chen, L. A Redox-Active 2D Metal-Organic Framework for Efficient Lithium Storage with Extraordinary High Capacity. Angew. Chem., Int. Ed. 2020, 59, 5273-5277.

(22) Matheu, R.; Gutierrez-Puebla, E.; Monge, M. A.; Diercks, C. S.; Kang, J.; Prévot, M. S.; Pei, X.; Hanikel, N.; Zhang, B.; Yang, P.; Yaghi, O. M. Three-Dimensional Phthalocyanine Metal-Catecholates for High Electrochemical Carbon Dioxide Reduction. J. Am. Chem. Soc. 2019, 141, 17081-17085.

(23) Park, J.; Hinckley, A. C.; Huang, Z.; Feng, D.; Yakovenko, A. A.; Lee, M.; Chen, S.; Zou, X.; Bao, Z. Synthetic Routes for a 2D Semiconductive Copper Hexahydroxybenzene Metal-Organic Framework. J. Am. Chem. Soc. 2018, 140, 14533-14537.

(24) Nagatomi, H.; Yanai, N.; Yamada, T.; Shiraishi, K.; Kimizuka, N. Synthesis and Electric Properties of a Two-Dimensional MetalOrganic Framework Based on Phthalocyanine. Chem. - Eur. J. 2018, 24, 1806-1810.

(25) Jia, H.; Yao, Y.; Zhao, J.; Gao, Y.; Luo, Z.; Du, P. A Novel TwoDimensional Nickel Phthalocyanine-Based Metal-Organic Framework for Highly Efficient Water Oxidation Catalysis. J. Mater. Chem. A 2018, 6, 1188-1195.

(26) Dou, J. H.; Sun, L.; Ge, Y.; Li, W.; Hendon, C. H.; Li, J.; Gul, S.; Yano, J.; Stach, E. A.; Dincă, M. Signature of Metallic Behavior in the Metal-Organic Frameworks $\mathrm{M}_{3}$ (Hexaiminobenzene $)_{2}(\mathrm{M}=\mathrm{Ni}$, Cu). J. Am. Chem. Soc. 2017, 139, 13608-13611.

(27) Skorupskii, G.; Trump, B. A.; Kasel, T. W.; Brown, C. M.; Hendon, C. H.; Dincă, M. Efficient and Tunable One-Dimensional Charge Transport in Layered Lanthanide Metal-Organic Frameworks. Nat. Chem. 2020, 12, 131-136.

(28) Furukawa, H.; Gándara, F.; Zhang, Y.-B.; Jiang, J.; Queen, W. L.; Hudson, M. R.; Yaghi, O. M. Water Adsorption in Porous MetalOrganic Frameworks and Related Materials. J. Am. Chem. Soc. 2014, $136,4369-4381$.

(29) Kalmutzki, M. J.; Hanikel, N.; Yaghi, O. M. Secondary Building Units as the Turning Point in the Development of the Reticular Chemistry of MOFs. Science Advances. 2018, 4, No. 10, eaat9180.

(30) Nguyen, N. T. T.; Furukawa, H.; Gándara, F.; Trickett, C. A.; Jeong, H. M.; Cordova, K. E.; Yaghi, O. M. Three-Dimensional Metal- 
Catecholate Frameworks and Their Ultrahigh Proton Conductivity. J. Am. Chem. Soc. 2015, 137, 15394-15397.

(31) Huang, J.; He, Y.; Yao, M.-S.; He, J.; Xu, G.; Zeller, M.; Xu, Z. A Semiconducting Gyroidal Metal-Sulfur Framework for Chemiresistive Sensing. J. Mater. Chem. A 2017, 5, 16139-16143.

(32) Ko, M.; Mendecki, L.; Mirica, K. A. Conductive TwoDimensional Metal-Organic Frameworks as Multifunctional Materials. Chem. Commun. 2018, 54, 7873-7891.

(33) Alexandropoulos, D. I.; Cunha-Silva, L.; Lorusso, G.; Evangelisti, M.; Tang, J.; Stamatatos, T. C. Dodecanuclear 3d/4fMetal Clusters with a 'Star of David' Topology: Single-Molecule Magnetism and Magnetocaloric Properties. Chem. Commun. 2016, 52, 1693-1696.

(34) Yaghi, O. M.; Kalmutzki, M. J.; Diercks, C. S. Introduction to Reticular Chemistry; Wiley, 2019.

(35) Day, R. W.; Bediako, D. K.; Rezaee, M.; Parent, L. R.; Skorupskii, G.; Arguilla, M. Q.; Hendon, C. H.; Stassen, I.; Gianneschi, N. C.; Kim, P.; Dincă, M. Single Crystals of Electrically Conductive Two-Dimensional Metal-Organic Frameworks: Structural and Electrical Transport Properties. ACS Cent. Sci. 2019, 5, 1959-1964.

(36) Sun, L.; Park, S. S.; Sheberla, D.; Dincǎ, M. Measuring and Reporting Electrical Conductivity in Metal-Organic Frameworks: $\mathrm{Cd}_{2}$ (TTFTB) as a Case Study. J. Am. Chem. Soc. 2016, 138, 1477214782.

(37) Brunauer, S.; Emmett, P. H.; Teller, E. Adsorption of Gases in Multimolecular Layers. J. Am. Chem. Soc. 1938, 60, 309-319.

(38) Suenaga, Y.; Inada, H.; Inomata, M.; Yamaguchi, R.; Okubo, T.; Maekawa, M.; Kuroda-Sowa, T. Crystal Structure and Characterization of Trinuclear Cobalt(III) Complex with 2,3,6,7,10,11Hexahydroxytriphenylene. Chem. Lett. 2014, 43, 562-564.

(39) Hoshino, N.; Akutagawa, T. A Trinuclear Iron(III) Complex of a Triple Noninnocent Ligand for Spin-Structured Molecular Conductors. Chem. - Eur. J. 2018, 24, 19323-19331. 